\title{
Therapeutic efficacy of chitosan nanoparticles loaded with BCG-polysaccharide nucleic acid and ovalbumin on airway inflammation in asthmatic mice
}

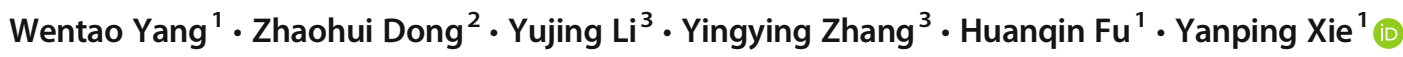

Received: 9 October 2020 / Accepted: 3 February 2021 / Published online: 5 March 2021

(C) The Author(s), under exclusive licence to Springer-Verlag GmbH Germany, part of Springer Nature 2021

\begin{abstract}
In this study, immunoregulation and desensitization therapies were jointly applied in the treatment of asthma, in which chitosan (CS) nanoparticles were used. BALB/c mice were selected and mouse models of asthma were constructed. Mice were divided into 7 groups. A double-chamber plethysmograph, MTT, hematoxylin-eosin staining, and ELISA were used. The expression levels of IL-4 and IL-5 in lung tissue cells were detected. CS-BCG-PSN-OVA sustained-release vaccines significantly alleviated airway hyperresponsiveness (AHR) in asthmatic mice. The numbers of total lymphocytes and eosinophils in BALF were remarkably reduced. The expression levels of IL-4 and IL-5 in lung tissue cells of the treatment groups were dramatically decreased. CS-BCG-PSN-OVA was found in vitro to be able to inhibit OVA-induced T-cell proliferation and upregulate the proportion of CD4+CD25+Foxp3+ T cells. CS-BCG-PSN-OVA sustained-release vaccine could significantly attenuate AHR and airway inflammation in asthmatic mice. Thus, it has a promising application prospect for the treatment of bronchial asthma.
\end{abstract}

Keywords Asthmatic mice $\cdot$ BCG-PSN $\cdot$ OVA $\cdot$ Chitosan $\cdot$ Sustained-release vaccine

\section{Introduction}

The main features of bronchial asthma are chronic airway inflammation, airway hyperresponsiveness (AHR), and reversible airflow limitation [1]. At present, it is believed that the overactivation of CD4+ T cells induced by allergen challenge leads to the imbalance of $\mathrm{Th} 1 / \mathrm{Th} 2$, in which Th2 cells were increased with the significantly increased Th2 cytokines, ultimately resulting in airway allergic inflammation dominated by infiltration of eosinophils [2,3]. Studies also indicated

Wentao Yang and Zhaohui Dong contributed equally to this work.

Yanping Xie

xieyp011@163.com

1 Department of Respiration, The First Hospital of Huzhou, The First Affiliated Hospital of Huzhou University, No.158 Guang Changhou Road, Huzhou 313000, Zhejiang, China

2 Department of Intensive Care Unit, The First Hospital of Huzhou, The First Affiliated Hospital of Huzhou University, Huzhou 313000, Zhejiang, China

3 School of Nursing, Huzhou University, Huzhou 313000, China that the decreased number and reduced function of CD4+ CD25+ regulatory $\mathrm{T}$ cells (Tregs) with inhibitory immune regulatory function lead to the overactivation of Tregs, consequently inducing the occurrence of asthma $[4,5]$. The pathogenesis of asthma is complex and has not been fully elucidated.

Main components of Bacillus Calmette-Guerin (BCG) include lipid, polysaccharides, proteins, and nucleic acids, among which polysaccharides and nucleic acids play an important role. BCG-polysaccharide nucleic acid (BCG-PSN) has been used in the clinical treatment of bronchial asthma with strong immunomodulatory effects. Studies suggested that BCG and BCG-PSN can inhibit the production of antigen-specific IgE, significantly reduce the airway eosinophil inflammation in asthmatic animals, and inhibit AHR [6, 7]. BCG is only used as an adjuvant drug clinically. Therefore, further improving the efficacy of BCG-PSN in the treatment of asthma may bring a better clinical application prospect.

Allergen-specific immunotherapy (desensitization) is also one of the effective methods for asthma treatment and its effectiveness has been clinically confirmed on patients with specific allergens. Desensitization therapy can relieve symptoms, reduce asthma deterioration, and attenuate AHR in asthmatic patients [8]. The present study considered that 
immunoregulation combined with desensitization therapy has better efficacy for asthma treatment than desensitization therapy alone and has less desensitization-related side effects [9].

Polysaccharide microparticles have become an important direction in the study of novel polysaccharide delivery systems [10-12], which contribute to improving the shortcomings of BCG preparations. BCG-PSN encapsulated by chitosan (CS) can protect the nucleic acid components from being destroyed by nuclease in vivo. Moreover, the polysaccharide components encapsulated by CS can form polysaccharide nanoparticles, which significantly enhance the bioavailability and have sustained release effect. In this study, immunoregulation and desensitization therapies were combined used in the treatment of asthma, with BCG-PSN and small-dose ovalbumin (OVA) encapsulated by CS nanoparticles simultaneously, so as to achieve better therapeutic effect.

\section{Materials and methods}

\section{Preparation of CS nanoparticles}

CS-BCG-PSN-OVA was prepared by ionic gelation method. CS was dissolved in $1 \%$ acetic acid solution to prepare a solution at a concentration of $1.5 \mathrm{mg} / \mathrm{mL}$ with the $\mathrm{pH}$ value of 5.5-5.7. Being stirred under continuous magnetic force, BCG-PSN and OVA were added to the CS solution, followed by stirring for $10 \mathrm{~min}$. Then, $0.1 \%$ sodium tripolyphosphate solution was added slowly until pale opalescent solution was formed. The size, dispersion, and state of CS nanoparticles were detected by a scanning electron microscope (JEOL, Akishima, Japan).

\section{Drug release in vitro}

To evaluate the in vitro release rate of CS particles, $10.0 \mathrm{mg}$ of particles was accurately weighed and dispersed in $5.0 \mathrm{~mL}$ phosphate-buffered saline (10 mM PBS, pH 7.3) containing $0.5 \%(\mathrm{w} / \mathrm{v})$ Tween 80 . Afterward, the solution was continuously cultured in a constant temperature oscillator $\left(37 \pm 1^{\circ} \mathrm{C}\right)$ at a rate of $80 \mathrm{r} / \mathrm{min}$. On days 1, 2, 3, 4, 5, and 6, $1.0 \mathrm{~mL}$ solution was collected and centrifuged at $6650 \times \mathrm{g}$ for $10 \mathrm{~min}$. The drug content in the supernatant was analyzed by highperformance liquid chromatography (HPLC).

\section{Establishment of asthmatic mouse models and drug administration}

Thirty-five female BALB/c mice (4-6 weeks old, weighed 16-22 g) were chosen and randomly divided into 7 groups (5 for each group) including CS-BCG-PSN-OVA group, BCG-PSN group, OVA group, BCG-PSN-OVA group, CS group, Normal group, and Model group. Except for the
Normal group, mice in the other groups were sensitized by intraperitoneal injection of $20 \mu \mathrm{g}$ OVA on days 0,7 , and 14, and then treated with 1\% OVA atomization inhalation for bronchial provocation on days 28, 29, 30, and 31 for once a day. The mice in the Normal group were treated by normal saline (NS) instead of OVA. Mice in each group except the Normal group and the CS group were intraperitoneally injected with corresponding drugs on days 18 and 24, while mice in the Normal group and CS group were given NS in a corresponding dose at indicated time points. The animal modeling intervention flow is detailed in Fig. 1.

\section{AHR detection}

A double-chamber plethysmograph (Buxco, Wilmington, NC, USA) was used for AHR detection. The mice were put into the chamber to monitor their respiration $48 \mathrm{~h}$ after the last provocation. Non-invasive lung function test was performed, and the enhanced pause (Penh) values were determined after methacholine $(\mathrm{Mch})$ provocation at increasing doses (3.12, $6.25,12.5,25,50$, and $100 \mathrm{mg} / \mathrm{mL}$ ). The Penh value determined after NS treatment was taken as the baseline value. The increase percentage of Penh (Penh/NS - 1) \% at different concentrations was calculated and used as a statistical indicator of airway responsiveness.

\section{Analysis of bronchoalveolar lavage fluid}

After the detection of AHR, bronchoalveolar lavage was performed by using PBS for 3 times. Then, the bronchoalveolar lavage fluid (BALF) was collected, centrifuged, and resuspended. Part of the suspension was taken for calculating the total number of cells. The remaining part was stained with hematoxylin-eosin (HE) for differential cell counts. There were 400 cells counted per slide and the number of different types of cells was recorded. The supernatant of BALF was retained to detect the levels of IL-4, IFN- $\gamma$, and IL- 10 .

\section{Enzyme-linked immunosorbent assay}

The contents of IL-4, IL-10, and IFN- $\gamma$ in BALF supernatant were determined by enzyme-linked immunosorbent assay (ELISA) according to the manufacturer's instructions. The diluted BALF was added into ELISA plate and incubated at $25^{\circ} \mathrm{C}$ for $2 \mathrm{~h}$, with the plate precoated with IL-4 (ab100710, abcam, China), IL-10 (ab100764, abcam, China), or IFN- $\gamma$ (ab46107, abcam, China) antibodies. The plate was washed and then added with conjugate for incubation at $25^{\circ} \mathrm{C}$ for $2 \mathrm{~h}$. After the wells were washed, BALF was incubated with substrate for $30 \mathrm{~min}$. The absorbance at $450 \mathrm{~nm}$ was measured by an ELISA reader. 
$1 \%$ ova atomization

inhalation excitation

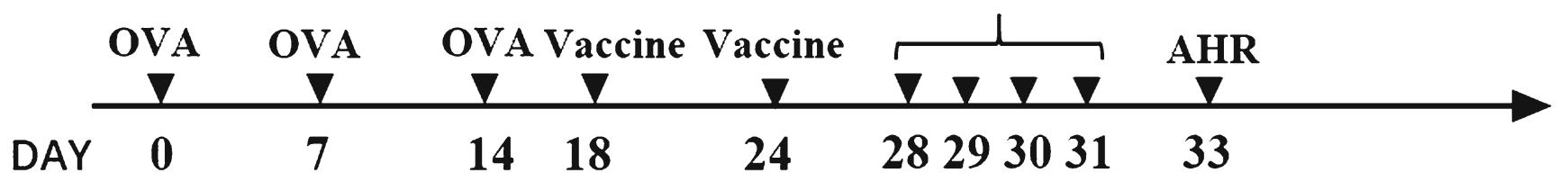

Fig. 1 The modeling process of asthmatic mouse models

\section{Histopathological examination}

The lungs were separated and inflated with $10 \%$ neutral formaldehyde. Then, the lungs were taken out and fixed in $10 \%$ neutral formaldehyde. The left lobe was removed, and treated with ethanol and xylene at gradient concentrations. Subsequently, it was paraffin embedded, sectioned, stained with $\mathrm{HE}$, and examined under a microscope.

\section{qRT-PCR}

Total RNA was extracted using Trizol (Invitrogen, CA, USA) according to the manufacturer's instructions. cDNA was synthesized using the reverse transcription system kit (Invitrogen, CA, USA). qRT-PCR was performed on the ABI 7900HT instrument (Applied Biosystems, USA) using SYBR Green assays (TaKaRa Biotechnology, Dalian, China). $\beta$-Actin was used as internal reference, and the difference in relative expression of target genes was calculated by $2^{-\Delta \Delta \mathrm{Ct}}$ method. The experiment was repeated three times. Primer sequences are listed in Table 1.

\section{Isolation of mice spleen-derived lymphocytes}

Mice were executed and immersed in alcohol for $5 \mathrm{~min}$. The skin of left abdomen was incised on a super clean bench, and the spleen was exposed. Then, the spleen was taken out, washed with $5 \mathrm{~mL}$ Hanks and transferred to a Petri dish with $4 \mathrm{~mL}$ mice spleen lymphocyte separation medium. Afterwards, the spleen was ground between the rough surfaces of two sterile slides within $5 \mathrm{~min}$, and the obtained cell suspension was softly treated with a pipette (not sharply), after

Table 1 Primer sequences used in qRT-PCR

\begin{tabular}{ll}
\hline Gene & Primer sequences \\
\hline IL-4 & F: 5'-ACA GGA GAA GGG ACG CCA T-3' \\
& R: 5'-GAA GCC GTA CAG ACG AGC TCA-3' \\
IL-5 & F: 5'-TGA GAC GAT GAG GCT TCC TG-3' \\
& R: 5'-ATTTCCACAGTACCCCCACG-3' \\
$\beta$-Actin & F: 5'-GCT TCT TTG CAG CTC CTT CGT-3' \\
& R: 5'-AGC GCA GCG ATA TCG TCA TC-3' \\
\hline
\end{tabular}

which the cell suspension was filtered through a 200-mesh nylon net and then transferred into a centrifugal tube. RPMI1640 of $1 \mathrm{~mL}$ was added to the centrifugal tube and centrifugation was run for $15 \mathrm{~min}$ at $2000 \mathrm{RPM}$. The cells in the lower liquid were the lymphocytes, which were absorbed by a pipette.

\section{MTT assay}

CS-BCG-PSN-OVA, BCG-PSN, OVA, and BCG-PSN-OVA were incubated with spleen-derived lymphocytes of asthmatic mice and OVA provocation was sequentially conducted. After being trypsinized and resuspended, cells were seeded in 96well plates at a density of $5 \times 10^{3}$ cells/well. After $72 \mathrm{~h}$ of culture, $10 \mu \mathrm{L}$ MTT reagent with the concentration of $5 \mathrm{mg} /$ $\mathrm{mL}$ was added for incubation at $37^{\circ} \mathrm{C}$ for $4 \mathrm{~h}$. The supernatant was discarded and sequentially $200 \mu \mathrm{L}$ dimethyl sulfoxide (DMSO) was added to the cells. The absorbance at $595 \mathrm{~nm}$ was measured by a microplate reader (Thermo Fisher Scientific).

\section{Analysis of Tregs}

CS-BCG-PSN-OVA, BCG-PSN, OVA, and BCG-PSNOVA were cultured with spleen-derived lymphocytes of asthmatic mice and then co-cultured with OVA. Cells were firstly incubated with antigen-presenting cell-labeled anti-CD4 and phycoerythrin (PE)-labeled anti-CD25 monoclonal antibodies (mAbs). Having been washed, cells were fixed and stained with Alexa Fluor 488conjugated anti-mouse Foxp3 mAbs or negative control mAbs. The cells were washed 3 times and resuspended in FCM buffer (PBS, $0.1 \%$ BSA and $0.1 \%$ NaN3). FASCalibur flow cytometry (Becton Dickinson, Franklin Lakes, NJ, USA) was used, and the data were analyzed using Cell-Quest software. Firstly, FSC/SSC was set as a gate to isolate lymphocytes. The CD4/CD25 gate was used. The percentage of Foxp3-stained CD4+CD25+ T cells was determined by the cell percentage of $\mathrm{T}$ cells stained with indicated anti-mouse mAbs minus that of the cells nonspecifically stained with negative control mAbs in the same dot-plot region. 


\section{Data statistics and analysis}

All data were processed by SPSS 21.0 statistical software (SPSS, Inc., Chicago, IL, USA), and the measurement data were expressed in the form of mean \pm standard deviation. The comparison between the two groups was analyzed by $t$-test, and one-way analysis of variance was used for comparison among multiple groups. Tukey's test was used for back testing. $P<0.05$ indicated a statistically significant difference.

\section{Results}

\section{Particle size of CS nanoparticles and in vitro release efficiency}

Firstly, we analyzed the CS-BCG-PSN-OVA nanoparticle by a scanning electron microscope. The result exhibited that the CS-BCG-PSN-OVA nanoparticle (Fig. 2B) was slightly larger than the empty CS particle (Fig. 2A), with an average particle size of $1130 \pm 22 \mathrm{~nm}$ and $923 \pm 17 \mathrm{~nm}$, respectively $(p<0.05)$.

Subsequently, in vitro release efficiency of the drug was tested. The cumulative release rate of the drug over time is showed in Fig. 2C. The in vitro release rates of BCG-PSN and OVA both exceeded $90 \%$ on day 6 . The above results indicated that the BCG-PSN and OVA could be slowly released in vitro after being encapsulated by CS nanoparticles, showing a better sustained release effect.

\section{CS-BCG-PSN-OVA significantly reduces airway responsiveness in asthmatic mice}

We conducted airway responsiveness test $48 \mathrm{~h}$ after the last provocation. The results indicated that compared with the Model group, the airway responsiveness of the mice in treatment groups (CS-BCG-PSN-OVA, BCG-PSN, OVA, BCGPSN-OVA) was significantly decreased upon the dose of Mch up to $12.5 \mathrm{mg} / \mathrm{mL}$, and the inhibitory effect remained with the increasing doses of Mch $(25 \mathrm{mg} / \mathrm{mL}, 50 \mathrm{mg} / \mathrm{mL}$, and $100 \mathrm{mg} /$
Fig. 2 Morphology of CS particles observed by a scanning electron microscope and in vitro release efficiency. (A) Empty CS particle. (B) CS-BCG-PSN-OVA particle. (C) In vitro release rate of BCG-PCN and OVA was detected by HPLC a

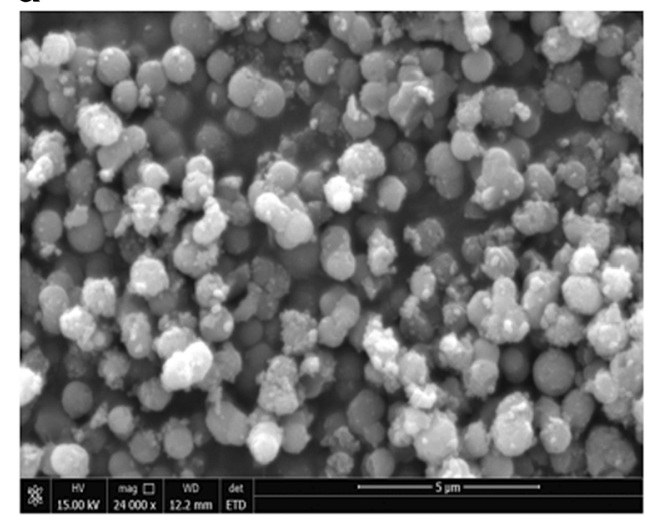

b

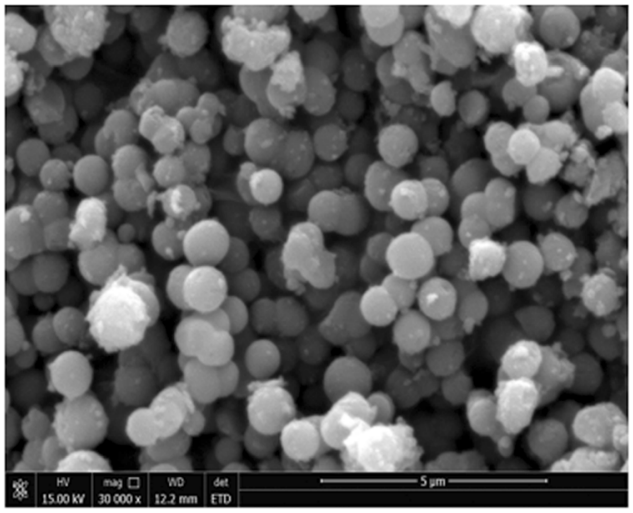

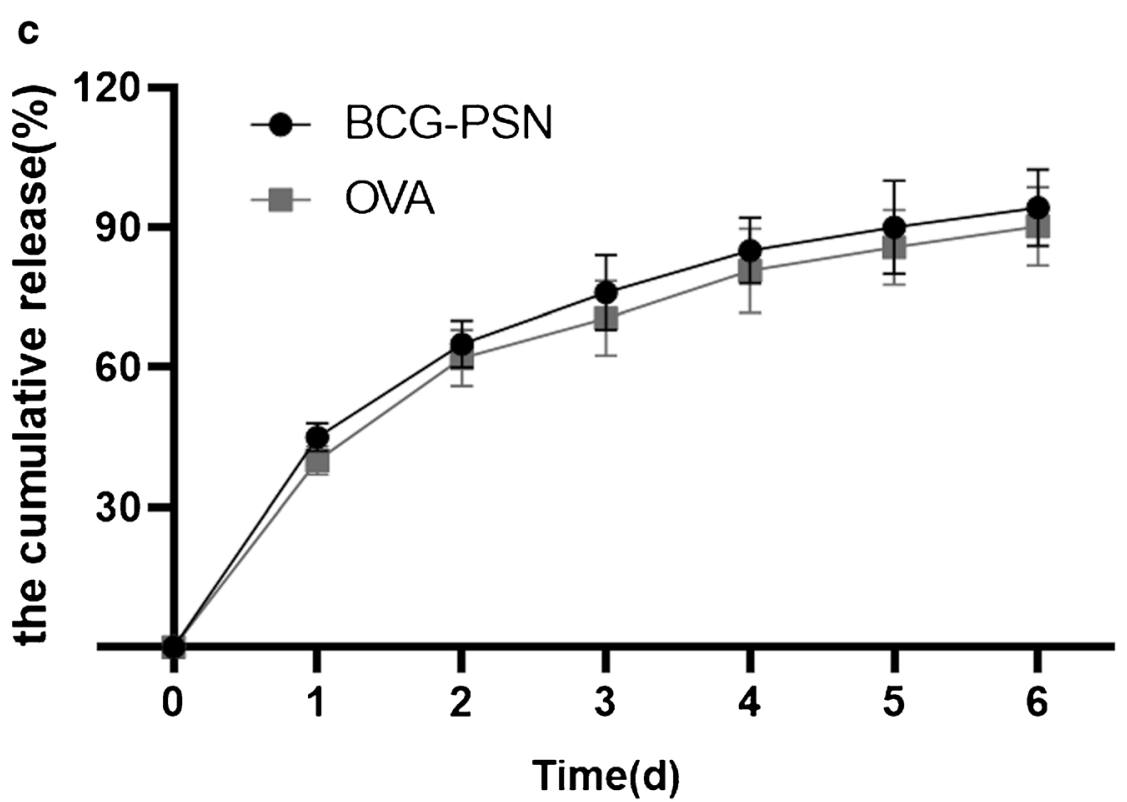


$\mathrm{mL})(p<0.05)$ (Fig. 3). Nevertheless, with a high dose of Mch $(50 \mathrm{mg} / \mathrm{mL}$ and $100 \mathrm{mg} / \mathrm{mL})$, the airway responsiveness in asthmatic mice treated with BCG-PSN or OVA alone was remarkably improved compared with that in mice treated with BCG-PSN-OVA $(p<0.05)$, which revealed that BCG-PSNOVS was more effective in reducing airway responsiveness in asthmatic mice. More notably, the airway responsiveness in mice was further alleviated after BCG-PSN-OVA was encapsulated with CS $(p<0.05)$, and the efficacy was the best among all the treatment groups. The above experimental results demonstrated that CS-BCG-PSN-OVA could significantly reduce the sensitivity of asthmatic mice to Mch, and the effect was better than that of unencapsulated drugs.

\section{CS-BCG-PSN-OVA significantly reduces airway inflammation in asthmatic mice}

To investigate whether CS-BCG-PSN-OVA could reduce airway inflammation in asthmatic mice, we counted the number of total lymphocytes and eosinophils in BALF. It was displayed that the total number of lymphocytes and eosinophils in each treatment group (including CS-BCG-PSN-OVA, BCG-PSN, OVA, and BCG-PSN-OVA) was obviously reduced compared with that in the Model group $(p<0.05)$, while there was no significant difference between the CS group and the Model group ( $p>0.05)$ (Table 2).

Then, the levels of IL- 4 , IFN- $\gamma$, and IL-10 in BALF supernatant were detected by ELISA (Fig. 4). Compared with the Model group, IL-4 was significantly reduced while IFN- $\gamma$, and IL-10 were dramatically increased in the treatment groups except the CS group ( $p<0.05)$. In addition, the lowest level of IL-4 and the highest levels of IFN- $\gamma$ and IL-10 were observed in the CS-BCG-PSN-OVA group. The above studies suggested that CS-BCG-PSN-OVA had a significant effect on reducing airway inflammation in mice.

\section{Lung histopathological changes in asthmatic mice after administration}

HE staining was used. It could be observed that under a light microscope, the alveolar wall in the normal group was complete and no exudate was in the alveolar cavity, with less infiltration of eosinophils and lymphocytes. While in the asthmatic groups and CS group, macrophage infiltration was observed in the alveolar cavity, with a large number of eosinophils around the trachea and blood vessels (Fig. 5A). After drug treatment in each group, infiltration of eosinophils and lymphocytes was significantly reduced $(p<0.05)$, and the therapeutic effect of the CS-BCG-PSN-OVA group was the best. Subsequently, the mRNA expression levels of IL-4 and IL-5 in lung tissue cells of each group were detected by qRT-PCR (Fig. 5B). Compared with the Model group, the expression levels of IL-4 and IL-5 in other treatment groups except the CS group were found to be significantly decreased, and those in the CS-BCG-PSN-OVA group were the lowest. These results indicated that CS-BCG-PSN-OVA significantly improved lung tissue lesions in asthmatic mice.

\section{The effect of CS-BCG-PSN-OVA on immune cells}

To investigate the effect of CS-BCG-PSN-OVA on cellular immune response, OVA was used, and the results were tested by MTT assay. Compared with the Model group, the proliferation activity of $\mathrm{T}$ cells in the treatment groups (CS-BCGPSN-OVA, BCG-PSN, OVA, BCG-PSN-OVA) was notably reduced, and the number of T cells in the CS-BCG-PSN-OVA group was the least (Table 3 ). Flow cytometry was used. The result showed that the proportion of $\mathrm{CD} 4+\mathrm{CD} 25+\mathrm{Foxp} 3+$ in spleen cells treated with drugs (CS-BCG-PSN-OVA, BCGPSN, OVA, BCG-PSN-OVA) was greatly higher than that in the Model group $(p<0.05)$. It was worth noting that the

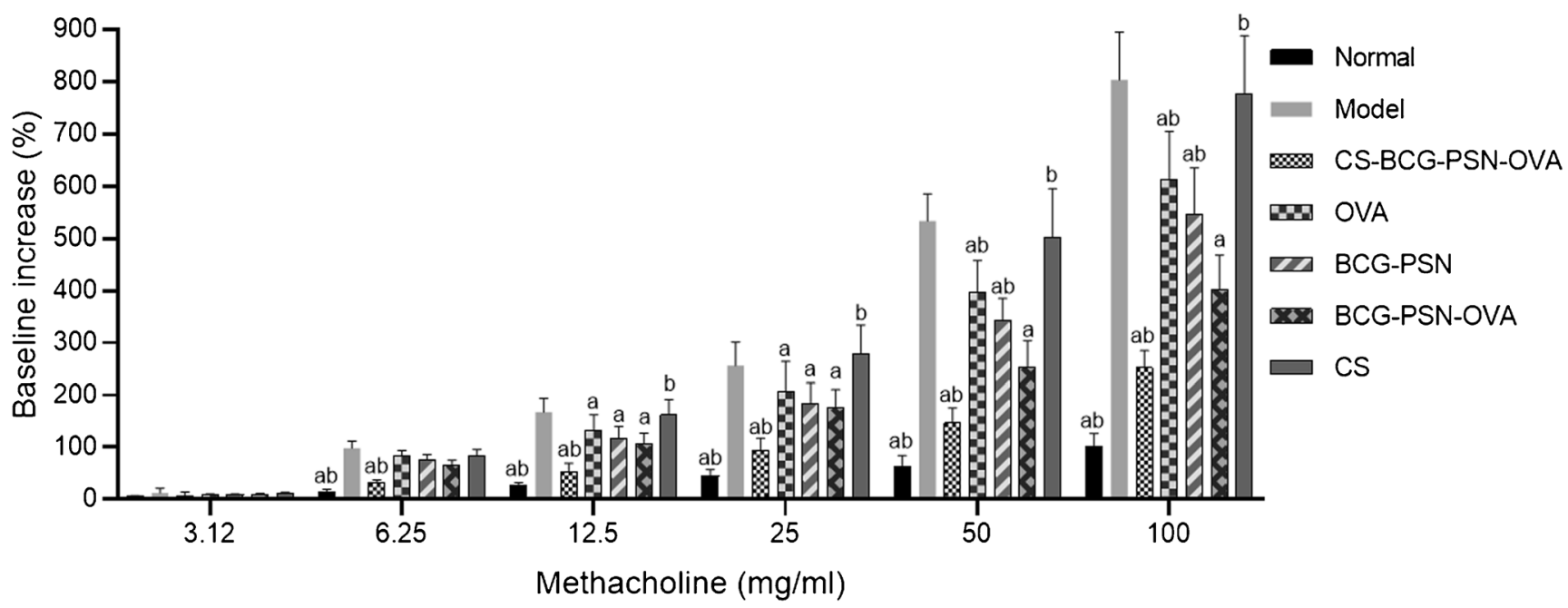

Fig. 3 Airway responsiveness in asthmatic mice of each group. "a" indicates a significant difference compared with the Model group $(p<0.05)$ and " $b$ " indicates that there is a significant difference compared with BCG-PSN-OVA group $(p<0.05)$ 
Table 2 Numbers of total cells and eosinophils in BALF

\begin{tabular}{|c|c|c|c|c|c|c|c|}
\hline Cells $\left(10^{4} / \mathrm{mL}\right)$ & Normal & Model & CS-BCG-PSN-OVA & OVA & BCG-PSN & BCG-PSN-OVA & $\mathrm{CS}$ \\
\hline Total cells & $39.36 \pm 5.11$ & $149.61 \pm 12.18$ & $69.21 \pm 6.19 * *$ & $119.11 \pm 8.35 *$ & $105.45 \pm 10.21 *$ & $84.61 \pm 10.42 * *$ & $142.64 \pm 13.14$ \\
\hline Eosinophils & 0 & $35.22 \pm 4.26$ & $15.12 \pm 2.16^{* *}$ & $28.33 \pm 3.26^{*}$ & $25.42 \pm 3.25^{*}$ & $19.22 \pm 1.72 * *$ & $33.23 \pm 5.18$ \\
\hline
\end{tabular}

${ }^{*} p<0.05$ compared with the Model group and $* * p<0.01$

proportion of CD4+CD25+Foxp3+ T cells was the most significantly increased after treatment with CS-BCG-PSN-OVA $(p<0.01)$ (Table 4$)$. These results suggested that CS-BCGPSN-OVA could effectively regulate the function of Tregs.

\section{Discussion}

BCG-PSN vaccine can significantly reduce airway inflammation and inhibit AHR in asthmatic animals [6]. Studies discovered that the eosinophilic airway inflammation was significantly inhibited in mice that received BCG treatment on days 1,7 , and 14 after birth, and IFN- $\gamma$ level in BALF and spleen cell suspension supernatant was upregulated, thereby activating Th1 cellular immune response and correcting Th1/Th2 imbalance $[13,14]$. These findings were further confirmed in our study. In our study, BCG-PSN vaccine was used in asthmatic mice and resulted in significant reduction of airway responsiveness and certain alleviation of airway inflammation.

A recent study reported that Th1 and Th2 cells, especially Tregs, are vital in the pathogenesis of asthma and allergies [15]. Our study found that BCG-PSN could regulate Tregs and significantly increase the proportion of CD4+CD25+Foxp3+. Although BCG-PSN shows a better therapeutic effect in animal experiments, it is only used as an adjuvant drug for the treatment of asthma in clinic, and no specific clinical data are available to illustrate its efficacy. However, it can be speculated that the clinical efficacy of BCG-PSN as an adjuvant drug may not be significant. Therefore, this study further explored a new treatment for bronchial asthma to improve its efficacy based on the above results.

We further included allergen-specific immunotherapy in this study. The mechanism of desensitization treatment has not been fully elucidated. Currently, it is believed that desensitization treatment can act on multiple different processes of asthma immune mechanism, including correcting Th1/Th2 imbalance, inducing the generation of blocking antibodies and Tregs, and inducing the allergen-specific immune tolerance of the body [16]. However, desensitization therapy is slow in efficacy, is long in duration, and requires gradual increase in the dosage of allergens, which may cause serious side effects on a few patients. Thus, it is only suitable for mild and moderate asthmatic patients [17]. Li and Liu et al. found that CS-encapsulated dust mites or main epitope-associated peptides can reduce airway responsiveness and airway inflammation in asthmatic mice by inducing the production of Tregs and stimulating Th1 response $[18,19]$. It is reported that the efficacy of immunoregulation combined with desensitization treatment for asthma is significantly better than that of desensitization treatment alone, and the desensitization-related side effects can be reduced [9]. Therefore, we jointly used BCGPSN and OVA to treat asthmatic mice. Under high doses of Mch $(25 \mathrm{mg} / \mathrm{mL}, 50 \mathrm{mg} / \mathrm{mL}$, and $100 \mathrm{mg} / \mathrm{mL})$, the BCGPSN-OVA complex medicine was more effective in reducing AHR and had better intervention effect on airway inflammation than BCG-PSN or OVA alone.

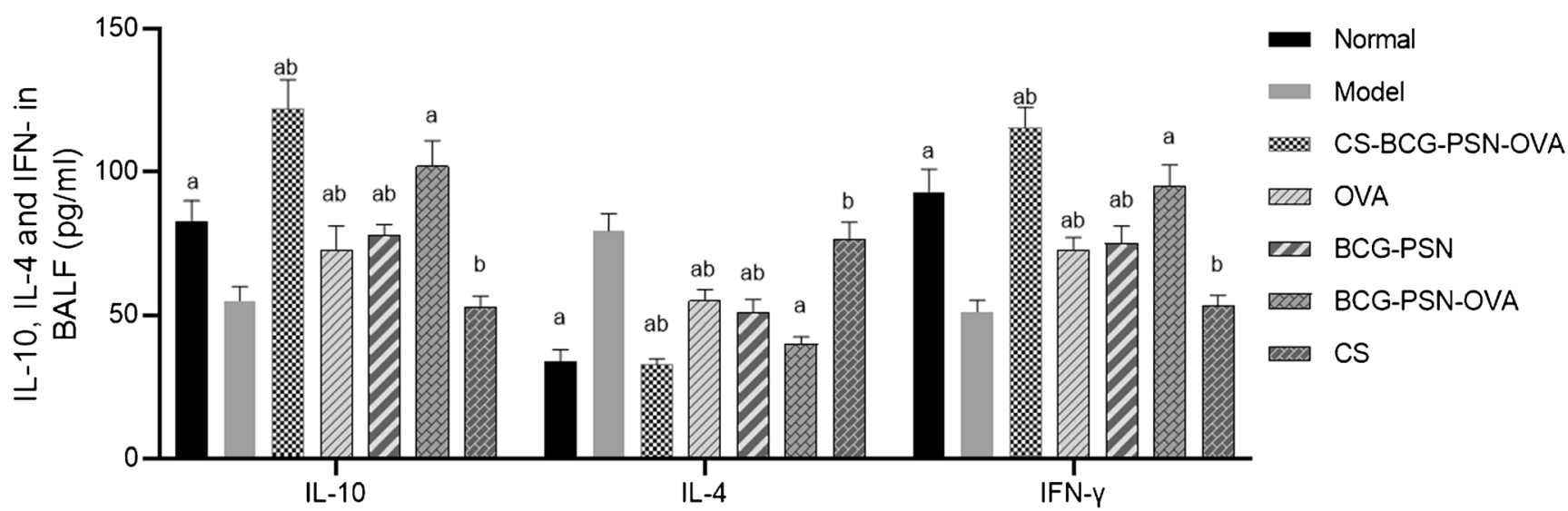

Fig. 4 Levels of IL-4, IFN- $\gamma$, and IL-10 in BALF supernatant of asthmatic mice in each group. "a" shows a significant difference compared with the Model group $(p<0.05)$. " $\mathrm{b}$ " indicates that there is a significant difference compared with the BCG-PSN-OVA group $(p<0.05)$ 

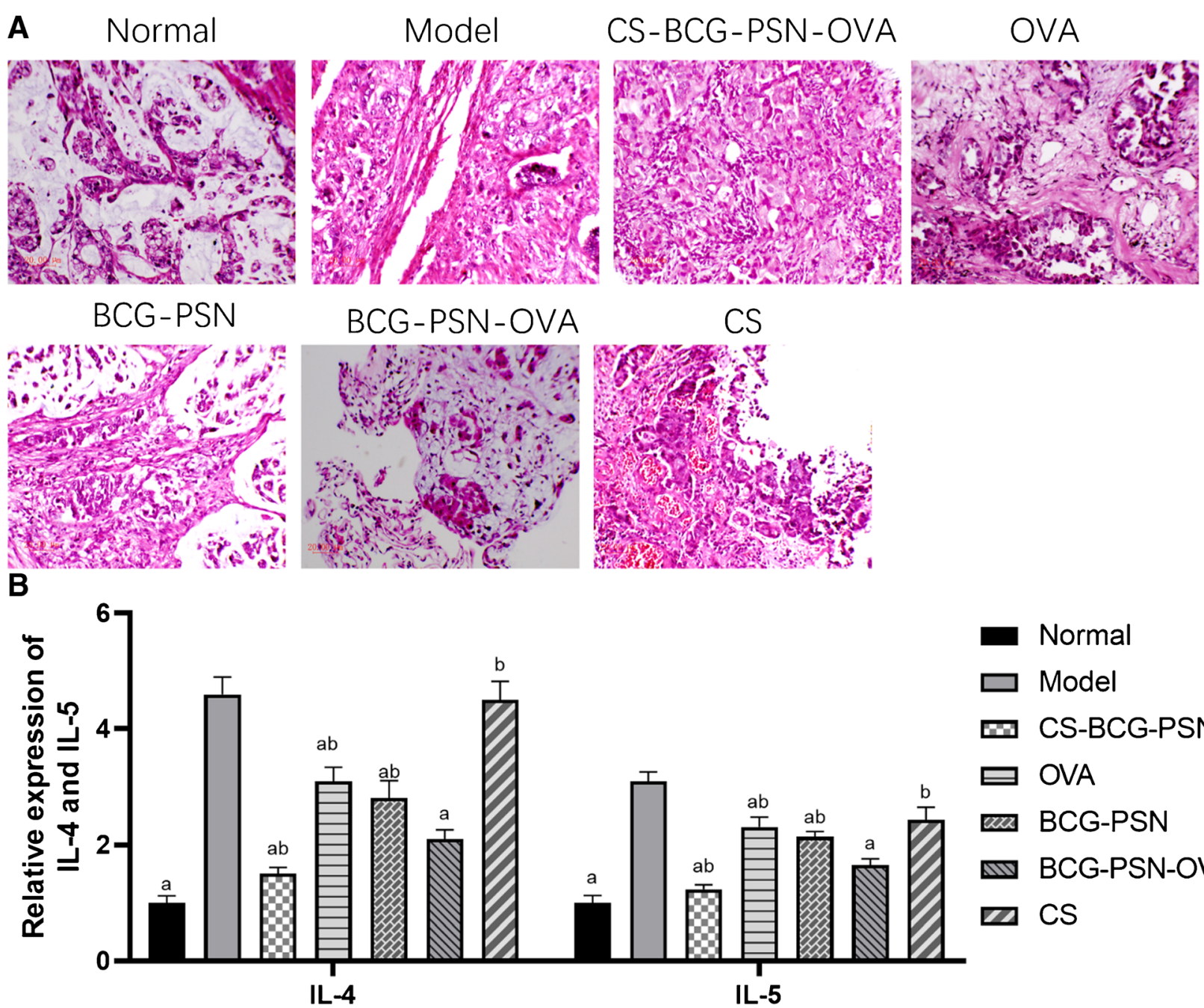

Fig. 5 Histopathological changes of lung tissue and expression of IL-4 and IL-5 in asthmatic mice after administration. (A) HE staining was used to observe the pathological change of lung tissue in asthmatic mice after administration. (B) The mRNA expression levels of IL-4 and IL-5 in lung

In the present study, BCG-PSN and OVA were encapsulated with CS nanoparticles to further improve the efficacy. CS is a biocompatible natural polysaccharide that can promote the transmembrane absorption of peptides and proteins, and plays a safe and effective role in allergen transmission $[18,20]$. CS microspheres are formed after nanocrystallization, and they can encapsulate nucleic acids, proteins and polysaccharide drugs, bases or nucleases in the body [21-23]. In our study, CS-BCG-PSNOVA sustained-release vaccine had the best effect on
Normal

$\square$ Model CS-BCG-PSN-OVA 曰 OVA एख BCG-PSN BCG-PSN-OVA $\square \mathrm{CS}$

Table 3 T cell proliferation in each group was detected by MTT assay after OVA stimulation

\begin{tabular}{llllllll}
\hline & Normal & Model & CS-BCG-PSN-OVA & OVA & BCG-PSN & BCG-PSN-OVA & CS \\
\hline OD $(595 \mathrm{~nm})$ & $0.15 \pm 0.02$ & $0.4 \pm 0.02$ & $0.19 \pm 0.03 * *$ & $0.23 \pm 0.01 *$ & $0.28 \pm 0.03 *$ & $0.21 \pm 0.01 *$ & $0.41 \pm 0.02$ \\
\hline
\end{tabular}

${ }^{*} p<0.05$ compared with the Model group and $* * p<0.01$ 
Table 4 The proportion of CD4+CD25+Foxp3+ in CD4+CD25+ T cells (\%)

\begin{tabular}{llllllll}
\hline & Normal & Model & CS-BCG-PSN-OVA & OVA & BCG-PSN & BCG-PSN-OVA & CS \\
\hline CD4+CD25+Foxp3+ (\%) & $36.16 \pm 4.11$ & $19.61 \pm 1.10$ & $39.21 \pm 2.29 * *$ & $25.11 \pm 2.15^{*}$ & $27.45 \pm 1.22 *$ & $34.61 \pm 1.34 *$ & $21.64 \pm 2.40$ \\
\hline
\end{tabular}

${ }^{*} p<0.05$ compared with the Model group and ${ }^{*} p<0.01$

In summary, our results confirmed that BCG-PSN-OVA was more effective in alleviating airway responsiveness and airway inflammation in asthmatic mice than BCG-PSN or OVA alone. Moreover, the CS-BCG-PSN-OVA sustainedrelease vaccine prepared by CS could further improve its efficacy. However, the specific mechanism and its clinical application need to be further clarified in future research.

Code availability Not applicable

Authors' contributions Wentao Yang contributed to the study design. Zhaohui Dong wrote the article. Yujing Li conducted the literature search. Yingying Zhang required the data. Huanqin Fu performed data analysis and drafted. Yanping Xie revised the article and gave the final approval of the version to be submitted.

Funding This project was supported by the Therapeutic efficacy of chitosan nanoparticles loaded with BCG-polysaccharide nucleic acid and ovalbumin on airway inflammation in asthmatic mice, the Public welfare application research project (2017GYB43) and Coronavirus pneumonia emergency prevention and control scientific research project (2020GYT17)

Data Availability The data and materials in this current study are available from the corresponding author on reasonable request.

\section{Declarations}

Ethics approval Not applicable

Consent to participate Not applicable

Consent to publication Not applicable

Conflict of interest The authors declare no competing interests.

\section{References}

1. Mims JW (2015) Asthma: definitions and pathophysiology. Int Forum Allergy Rhinol 5(Suppl 1):S2-S6. https://doi.org/10.1002/ alr.21609

2. Harb H, Renz H (2015) Update on epigenetics in allergic disease. J Allergy Clin Immunol 135:15-24. https://doi.org/10.1016/j.jaci. 2014.11.009

3. Umetsu DT, McIntire JJ, Akbari O, Macaubas C, DeKruyff RH (2002) Asthma: an epidemic of dysregulated immunity. Nat Immunol 3:715-720. https://doi.org/10.1038/ni0802-715
4. Cohn L, Elias JA, Chupp GL (2004) Asthma: mechanisms of disease persistence and progression. Annu Rev Immunol 22:789-815. https://doi.org/10.1146/annurev.immunol.22.012703.104716

5. Apter AJ (2015) Advances in adult asthma diagnosis and treatment in 2014. J Allergy Clin Immunol 135:46-53. https://doi.org/10. 1016/j.jaci.2014.10.050

6. Han ER, Choi IS, Eom SH, Kim HJ (2010) Preventive effects of mycobacteria and their culture supernatants against asthma development in BALB/c mice. Allergy, Asthma Immunol Res 2:34-40. https://doi.org/10.4168/aair.2010.2.1.34

7. Christ AP, Rodriguez D, Bortolatto J, Borducchi E, Keller A, Mucida D, Silva JS, Leite LC, Russo M (2010) Enhancement of Th1 lung immunity induced by recombinant Mycobacterium bovis Bacillus Calmette-Guerin attenuates airway allergic disease. Am J Respir Cell Mol Biol 43:243-252. https://doi.org/10.1165/rcmb. 2009-0040OC

8. Frew AJ (2010) Allergen immunotherapy. J Allergy Clin Immunol 125:S306-S313. https://doi.org/10.1016/j.jaci.2009.10.064

9. Kopp MV (2011) Role of immunmodulators in allergen-specific immunotherapy. Allergy 66:792-797. https://doi.org/10.1111/j. 1398-9995.2011.02553.x

10. Zhang L, Yang L, Zhang X, Jiaqi L, Fan L, Beck-Broichsitter M, Zhang X, Muenster U, Wang X, Zhao J, Zhang Y, Mao S (2018) Sustained therapeutic efficacy of budesonide-loaded chitosan swellable microparticles after lung delivery: Influence of in vitro release, treatment interval and dose. J Control Release 283:163174. https://doi.org/10.1016/j.jconrel.2018.05.031

11. Duttagupta DS, Jadhav VM, Kadam VJ (2015) Chitosan: a propitious biopolymer for drug delivery. Curr Drug Deliv 12:369-381. https://doi.org/10.2174/1567201812666150310151657

12. Kolawole OM, Lau WM, Khutoryanskiy VV (2018) Methacrylated chitosan as a polymer with enhanced mucoadhesive properties for transmucosal drug delivery. Int J Pharm 550:123-129. https://doi. org/10.1016/j.ijpharm.2018.08.034

13. Shen H, Huang H, Wang J, Ye S, Li W, Wang K, Zhang G, Wang P (2008) Neonatal vaccination with Bacillus Calmette-Guérin elicits long-term protection in mouse-allergic responses. Allergy 63:555563. https://doi.org/10.1111/j.1398-9995.2008.01637.x

14. Zhang G, Wang P, Qiu Z, Qin X, Lin X, Li N, Huang H, Liu H, Hua W, Chen Z, Zhao H, Li W, Shen H (2013) Distant lymph nodes serve as pools of Th1 cells induced by neonatal BCG vaccination for the prevention of asthma in mice. Allergy 68:330-338. https:// doi.org/10.1111/all.12099

15. Akdis M, Blaser K, Akdis CA (2005) T regulatory cells in allergy: novel concepts in the pathogenesis, prevention, and treatment of allergic diseases. J Allergy Clin Immunol 116:961-968; quiz 969. https://doi.org/10.1016/j.jaci.2005.09.004

16. Pipet A, Botturi K, Pinot D, Vervloet D, Magnan A (2009) Allergen-specific immunotherapy in allergic rhinitis and asthma. Mechanisms and proof of efficacy. Respir Med 103:800-812. https://doi.org/10.1016/j.rmed.2009.01.008

17. Spertini F, Reymond C, Leimgruber A (2009) Allergen-specific immunotherapy of allergy and asthma: current and future trends. Exp Rev Respir Med 3:37-51. https://doi.org/10.1586/17476348.3. 1.37 
18. Li J, Liu Z, Wu Y, Wu H, Ran P (2008) Chitosan microparticles loaded with mite group 2 allergen Der $\mathrm{f} 2$ alleviate asthma in mice. $\mathrm{J}$ Investig Allergol Clin Immunol 18:454 460

19. Liu Z, Guo H, Wu Y, Yu H, Yang H, Li J (2009) Local nasal immunotherapy: efficacy of Dermatophagoides farinae-chitosan vaccine in murine asthma. Int Arch Allergy Immunol 150:221228. https://doi.org/10.1159/000222674

20. Jabbal-Gill I, Fisher AN, Rappuoli R, Davis SS, Illum L (1998) Stimulation of mucosal and systemic antibody responses against Bordetella pertussis filamentous haemagglutinin and recombinant pertussis toxin after nasal administration with chitosan in mice. Vaccine 16:2039-2046. https://doi.org/10.1016/s0264-410x(98) 00077-2
21. Mao S, Sun W, Kissel T (2010) Chitosan-based formulations for delivery of DNA and siRNA. Adv Drug Deliv Rev 62:12-27. https://doi.org/10.1016/j.addr.2009.08.004

22. Kravanja G, Primožič M, Knez Ž, Leitgeb M (2019) Chitosanbased (Nano)materials for Novel Biomedical Applications. Molecules (Basel, Switzerland) 24. https://doi.org/10.3390/ molecules24101960

23. Kamath PR, Sunil D (2017) Nano-Chitosan Particles in Anticancer Drug Delivery: An Up-to-Date Review. Mini-Rev Med Chem 17: 1457-1487. https://doi.org/10.2174/1389557517666170228105731

Publisher's note Springer Nature remains neutral with regard to jurisdictional claims in published maps and institutional affiliations. 